\title{
Biomedicalización e infancia: \\ trastorno de déficit de atención e hiperactividad
}

Celia Iriart ${ }^{1}$

Lisbeth Iglesias Ríos²

IRIART, C.; IGLESIAS RÍOS, L. Biomedicalization and childhood: attention deficit hyperactivity disorder. Interface - Comunic., Saude, Educ., v.16, n.43, p.1011-23, out./dez. 2012.

This article critically analyzes the increasing number of children diagnosed with and treated for Attention Deficit Hyperactivity Disorder (ADHD). The analysis links this growing phenomenon with the strategies of the pharmaceutical industry for attaining leadership in conceptualizing the health-illness-care process as well as in the healthcare market. We utilized analytical and interpretive methods to study primary and secondary data and conducted an extensive literature review. In the light of the concept of biomedicalization, we analyzed the ideological and subjective mechanisms that facilitated institutionalization of this discourse as a new truth concerning this disease as well as its legitimization by governmental and civic organizations. The biomedicalization of children's distress facilitates the concealment of deeply rooted socioeconomic, political, ideological and cultural changes that have radically transformed our societies over the past few decades.

Keywords: Attention Deficit Hyperactivity Disorder (ADHD). Biomedicalization. Healthcare consumer. DSM. Medical industrial complex.
El artículo analiza críticamente el aumento de los niños diagnosticados y tratados por el Trastorno de Déficit de Atención e Hiperactividad (TDAH). Los análisis vinculan este creciente fenómeno con las estrategias de la industria farmacéutica para reposicionarse en el liderazgo de la conceptualización del proceso saludenfermedad-atención y en el mercado de salud. Utilizamos métodos analíticointerpretativos para estudiar datos primarios y secundarios, y realizar una extensa revisión bibliográfica. A la luz del concepto de biomedicalización analizamos los mecanismos subjetivo-ideológicos que facilitaron que este discurso se instituya como una nueva verdad sobre este trastorno y sea legitimado por los organismos gubernamentales y las organizaciones de la sociedad civil. La biomedicalización del sufrimiento infantil dificulta que se pongan en evidencia los profundos cambios socioeconómicos, políticos e ideológico-culturales que han transformado radicalmente nuestras sociedades en las últimas décadas.

Palabras clave: Trastorno por Déficit de Atención con Hiperactividad (TDAH). Biomedicalización. Consumidor de salud. DSM. Complejo médico-industrial. Utilización de medicamentos.

\footnotetext{
${ }^{1}$ Department of Family and Community Medicine, Public Health Program, University of New Mexico. MSC09 5060, 1 University of New Mexico, Albuquerque, NM 87131-0001, USA ciriart@salud.unm.edu ${ }^{2}$ Public Health Program, University of New
} 


\section{Presentación del problema}

Si bien el TDAH es un trastorno definido hace varias décadas, su más reciente redefinición y la extensa difusión de la que está siendo objeto desde finales de la década del noventa nos indica un cambio que requiere ser analizado. De acuerdo a documentos oficiales y artículos científicos, la cantidad de niños diagnosticados y tratados con el Trastorno por Déficit de Atención con o sin Hiperactividad (TDAH) aumentó considerablemente en los países latinoamericanos desde comienzos de la década del 2000, con un acentuado crecimiento hacia finales de la misma (Ortega et al., 2010; Frenk Mora et al., 2002). Estos datos de aumento en la prevalencia del TDAH no se basan en investigaciones epidemiológicas nacionales en países latinoamericanos, sino en estudios parciales generalmente basados en casos clínicos, en extrapolaciones de las prevalencias publicadas en la bibliografía anglosajona, o en datos de aumento del consumo de las drogas usadas para tratarlo. Este proceso acompaña la tendencia que se consolidó en los Estados Unidos, país donde primero se difunde la idea de que el TDAH está subdiagnosticado (Center for Disease Control and Prevention, 2011).

El aumento de niños diagnosticados parece correlacionarse con las estrategias que el complejo médico-industrial, especialmente la industria farmacéutica, generó para contrarrestar las reformas que el capital financiero desarrolló, a partir de la década del noventa, con su entrada como administrador de programas de salud y planes de cobertura de atención médica. Estas reformas se desarrollaron primero en los Estados Unidos y luego se exportaron a numerosos países de América Latina, Asia, y Europa. Ambos procesos han sido extensamente estudiados por Iriart junto a otros colegas (Iriart, Franco, Merhy, 2011; Iriart, Merhy, Waitzkin, 2000). Aquí presentamos una breve síntesis basada en esas investigaciones para contextualizar los análisis relacionados con el TDAH.

Durante las décadas previas a la implantación de las reformas sectoriales bajo la hegemonía de los grupos financieros, la industria farmacéutica dirigió sus estrategias promocionales a los médicos. Así fue como la utilización de servicios y productos biomédicos estuvo determinada en gran medida por la oferta de los mismos a través de estos profesionales. La comprensión de esta situación por parte de los grupos financieros que instalaron las reformas denominadas de atención gerenciada, generó estrategias de contención de gastos basadas en el control del uso de servicios y tratamientos. A partir de estos cambios en la administración de coberturas médicas, el complejo médico-industrial, especialmente la industria farmacéutica, inició una serie de estrategias para restablecer su liderazgo en la conceptualización del proceso salud-enfermedad-atención y en el mercado de salud. Una de las estrategias estuvo centrada en radicalizar a su favor el concepto de consumidor que las reformas basadas en la atención gerenciada habían cooptado de sus promotores iniciales, los grupos que cuestionaban el poder médico en los setentas (Critser, 2005). Con el objeto de utilizar para sus fines comerciales el concepto de consumidor, la industria farmacéutica utilizó diversos mecanismos para establecer una relación directa con los potenciales usuarios de sus productos que analizaremos en la siguiente sección.

Sin embargo, como veremos las estrategias de la industria farmacéutica para dirigir sus mensajes al público tienen implicaciones mucho más profundas en nuestras sociedades ya que se inscriben en procesos de creación de nuevas subjetividades que radicalizan la medicalización, y que siguiendo a otros autores vamos a denominar biomedicalización (Clarke et al., 2010). Consideramos que el concepto de biomedicalización ofrece una mejor interpretación de los profundos cambios operados en la década del noventa en la conceptualización del proceso salud-enfermedad-atención. La medicalización implica la expansión del diagnóstico y tratamiento médico de situaciones previamente no consideradas problemas de salud, como por ejemplo, la reproducción humana. La biomedicalización, por su parte, supone la internalización de la necesidad de autocontrol y vigilancia por parte de los individuos mismos, no requiriendo necesariamente la intervención médica. No se trata, solamente, de definir, detectar y tratar procesos mórbidos, sino de estar alerta de potenciales riesgos e indicios que pueden derivar en una patología. En el caso del TDAH, por ejemplo, la disponibilidad de sencillos métodos de diagnóstico y la extensa información en internet y otros medios puestos al servicio de padres, maestros y profesionales no especializados facilita la difusión del mismo, el autocontrol y la vigilancia.

En base a lo previamente introducido, el objetivo del artículo es aportar una lectura crítica desde la salud colectiva sobre el TDAH contextualizándolo en los procesos antes descriptos. Para ello, primero 
sintetizaremos los principales elementos de la reforma relacionados con el tema, luego examinaremos el concepto de biomedicalización en relación al TDAH, posteriormente presentaremos una reflexión crítica acerca de la construcción del TDAH como un problema de salud pública y, finalmente, analizaremos los cambios en las definiciones diagnósticas y las diferentes clasificaciones utilizadas que determinan el número de niños incluidos en este diagnóstico.

\section{Método}

Para esta investigación usamos metodología analítica para reinterpretar datos secundarios e investigaciones desarrollados por otros autores que analizan el aumento de la prevalencia de niños diagnosticados con TDAH en las últimas dos décadas. Partimos de la premisa de la existencia de una anomalía en la forma en que se define, cuantifica y trata este problema desde las corrientes hegemónicas de la psiquiatría basadas en concepciones neurobiológicas. Sin esto no es posible explicar el aumento de niños diagnosticados y tratados por este trastorno en un corto período de tiempo en el cual no han surgido nuevos métodos diagnósticos considerados "objetivos", tal el caso de marcadores bioquímicos, pruebas neuropsicológicas o genéticas, o estudios de neuroimágenes

Recurrimos a fuentes primarias de información tales como portales en la red y blogs para ejemplificar la información que el público recibe y los debates existentes. Los estudios de los que participara una de las autoras (Iriart) fueron utilizados para contextualizar los análisis sobre el TDAH en los procesos de reforma sectorial. Revisamos las bases de datos Scielo, PubMed, JSTOR y PsycINFO cubriendo el período enero 1990 a mayo 2011 usando los siguientes términos en español, inglés y portugués: "trastorno por déficit de atención e hiperactividad (TDAH)", "DSM", "Clasificacion Internacional de Enfermedades (CIE)", "industria farmacéutica", "mercadotecnia", y "medicalización". También usamos como fuente la literatura citada por los autores analizados cuando consideramos que era útil para profundizar el análisis. Sin embargo, queremos destacar que este artículo no usa la metodología de revisión sistemática, por lo cual seleccionamos aquellas fuentes que aportaban al análisis que nos proponemos desarrollar en este artículo. Por ello la lista de referencias en el artículo incluye solo las más relevantes de la extensa revisión realizada, cuidando de incluir autores de diversos campos y las más recientes en cada tema descripto. Usamos la revisión bibliográfica para seleccionar materiales que describen el proceso histórico, político y económico que permitió dar mayor visibilidad al trastorno, y para describir cómo, en la actualidad, se están usando los medios de comunicación por parte de grupos no profesionales, como así también por organismos gubernamentales, y compañías farmacéuticas para expandir el conocimiento sobre este trastorno y proveer instrumentos no científicos a la población para realizar un primer diagnóstico del problema. Asimismo, la revisión bibliográfica nos permitió analizar los cambios en la definición del TDAH y las diferencias en la prevalencia según la clasificación usada. Analizamos críticamente los datos de prevalencia oficialmente publicados en los Estados Unidos por ser la fuente más utilizada por los medios de comunicación y por los investigadores al informar sobre la prevalencia del trastorno también en los países latinoamericanos.

\section{Biomedicalización de la infancia y TDAH}

La transformación de la medicalización en biomedicalización fue posible por la confluencia de diferentes aspectos entre los cuales destacamos la masiva entrada del capital financiero en el sector salud y los reacomodamientos que el complejo médico-industrial realizó para recuperar liderazgo ante esta nueva situación. Estos procesos aumentaron exponencialmente la privatización del sector salud en manos de corporaciones nacionales o multinacionales, así como la masiva mercantilización de sus productos y servicios (Iriart, Franco, Merhy, 2011). En el caso de enfermedades y trastornos ya conocidos y medicalizados como el TDAH, lo que las farmacéuticas hicieron fue expandir el mercado desarrollando mecanismos comunicacionales para que se internalice el problema como un trastorno subdiagnosticado y que puede ser controlado con fármacos. Estos procesos fueron favorecidos por la transformación del 
modelo de negocios que la industria farmacéutica desarrolló. La industria pasó de un modelo centrado en la "educación" de los profesionales de salud, en especial los médicos, para que prescriban sus productos, a otro en que el consumidor directo ocupa un papel central en las campañas de comercialización (Iriart, Franco, Merhy, 2011). Estas campañas de comercialización pueden ser explícitas como en los Estados Unidos, donde los medicamentos bajo prescripción pueden ser publicitados a través de la prensa escrita y oral; o encubiertas como en los países latinoamericanos, donde la legislación para la publicidad de medicamentos bajo prescripción lo impide. En los países latinoamericanos hemos observado la utilización de las campañas de concientización de enfermedades usando los medios masivos de difusión pero sin nombrar la medicación, y presentaciones en ámbitos educativos o en programas de radio y televisión donde "expertos" en el tema "educan" a la audiencia para que sean capaces de identificar los síntomas del TDAH. El internet se ha convertido en otra herramienta útil, ya que ofrece fácil acceso a incontables espacios dedicados a la identificación y tratamiento del TDAH incluyendo sencillos cuestionarios para detectar los síntomas (Associação Brasileira do Déficit de Atenção, 2011; American Academy of Pediatrics, 2010)³. Estos espacios de información son mantenidos inclusive por organizaciones gubernamentales como los Centros para el Control de Enfermedades y Prevención (CDC, por su sigla en inglés). Desde su sitio, el CDC ofrece a los usuarios, incluyendo a los hispanohablantes, suscribirse para recibir noticias y actualizaciones sobre el TDAH, y el acceso a herramientas comunicacionales para difundir información entre familiares y amigos (CDC, 2011). La industria farmacéutica también brinda apoyo financiero a asociaciones de pacientes para que difundan el trastorno y sus posibles tratamientos a través de sus sitios en la red (Moynihan, Cassels, 2005).

Estos mecanismos comunicacionales han facilitado la redefinición de la medicalización como biomedicalización (Clarke et al., 2010). El concepto de medicalización fue descrito por varios autores en los setentas como la definición y captura, para fines diagnósticos y de tratamiento médico, de aspectos de la vida previamente fuera de la jurisdicción médica (Illich, 1975). Para entender las transformaciones operadas en décadas recientes, Clarke y colegas desarrollaron el concepto de biomedicalización basándose en la teoría foucaultiana del biopoder y en desarrollos más reciente realizados por Rabinow (1995). Mientras la medicalización se centra en el padecimiento, la enfermedad, el cuidado y la rehabilitación; la biomedicalización se enfoca en la salud como un mandato moral que internaliza el auto control, la vigilancia y la transformación personal. La biomedicalización implica un cambio en relación al concepto de medicalización, en tanto se pasa de un creciente control de la naturaleza (el mundo alrededor del sujeto) a la internalización del control y transformación del propio sujeto y su entorno, transformando la vida misma. La disponibilidad y masiva accesibilidad a tecnologías biológicas, incluyendo medicamentos, instrumentos diagnósticos y otros equipamientos, así como el acceso a enormes cantidades de información sobre enfermedades y trastornos (nuevos, viejos y redefinidos), crean nuevas subjetividades, identidades, y biosocialidades.

En el caso del TDHA una nueva subjetividad emerge cuando se identifica al niño inquieto $\mathrm{y} / \mathrm{o}$ distraído como portador de un trastorno, ya no como un niño travieso, o con necesidades diferentes, o que está llamando la atención sobre situaciones que lo afectan (Miller, 1999). Las relaciones sociales se transforman y el niño pasa a ser etiquetado como un niño con una discapacidad para el aprendizaje o la sociabilidad. Esto es internalizado por el niño, la familia, los amigos y los maestros, creándose así una nueva identidad en torno al

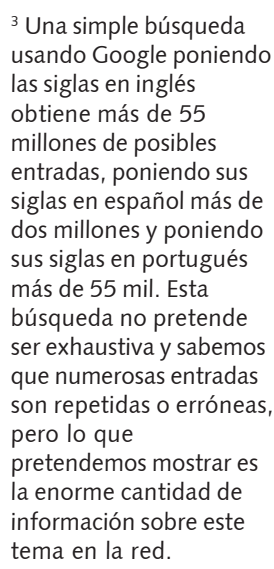


padecimiento. A su vez, la existencia de grupos informales y de asociaciones de pacientes o familiares con el trastorno posibilita nuevas formas de sociabilidad que están determinadas por el padecimiento, de ahí que las denominemos biosociabilidades. Internet ofrece una gran oferta de herramientas, tales como, portales, redes sociales (facebook, twitter) y blogs, para intercambiar o debatir acerca de temas específicos como el TDHA. Estos fórums en la red están muchas veces promovidos por la industria farmacéutica o por asociaciones de pacientes o profesionales (Children and Adults With AttentionDeficit/Hyperactivity Disorder, 2011). Sin embargo, es importante destacar que no todas estas nuevas identidades implican la aceptación de los discursos y las prácticas biomedicalizadoras. Algunos de estos grupos/fórums cuestionan el mandato moral y otras formas de biomedicalización (Death from Ritalin, 2011; The Natural Child Project, 2011). El proceso no es unidireccional y diferentes tipos de discursos son creados por una multiplicidad de individuos y organizaciones. Sin embargo, al identificarse en base a la biosocialidad creada alrededor del TDHA o los medicamentos utilizados, estos grupos si bien con un mensaje cuestionador, no escapan a esta construcción de agrupamientos biosociales, que, como señala Ortega (2004), sustituyen progresivamente los agrupamientos tradicionales de clase, religión u orientación política.

Nuevos desarrollos organizacionales y regulaciones pueden cambiar la situación que, en la actualidad, hegemoniza el complejo médico-industrial en torno a procesos de salud-enfermedad. Más acceso a información sobre temas de salud favorece la democratización de este campo, el problema es qué tipo de información se trasmite, quién genera los datos, cómo se construyen y difunden los mismos, y qué intereses movilizan su circulación. La diseminación descontextualizada de información sobre salud reproduce la biomedicalización y genera un sentido común en el cual, en el caso del TDAH, padres y maestros se ven compelidos a actuar según esta ideología de control individual. Esto genera una profunda mercantilización del sufrimiento infantil, ya que los padres, convertidos en consumidores, buscarán los servicios de los profesionales recomendados en los sitios, o si recurren a otros, irán muñidos de información que les permitirá describir más precisamente signos y síntomas conducentes al diagnóstico del TDAH y posiblemente a la medicación.

En esta sección ubicamos el problema de la creciente difusión del TDHA en los procesos estructurales que crearon las condiciones de posibilidad para que emerja un nuevo discurso sobre este trastorno y analizamos los procesos de subjetivación que la biomedicalización genera. A continuación analizaremos cómo los datos son producidos, interpretados y difundidos, facilitando que se instituya una nueva verdad sobre el TDHA, y sean utilizados para legitimar los discursos biomedicalizadores por parte de organismos gubernamentales y organizaciones de la sociedad civil.

\section{La construcción del TDAH como un problema de salud pública}

Según las definiciones más recientes, el TDAH es un trastorno psiquiátrico neuro-conductual que se caracteriza por el desarrollo de niveles inapropiados de inatención y/o hiperactividad, los que resultan en incapacidad de funcionar adecuadamente en espacios escolares, familiares y sociales (CDC, 2011). La forma de diagnóstico más difundido se basa en la detección de los siguientes síntomas: hiperactividad, falta de atención e impulsividad. El diagnóstico del TDAH se realiza solo en base a observaciones del comportamiento de los niños, ya que no existen pruebas definidas como objetivas (marcadores bioquímicos, pruebas neuropsicológicas o genéticas, o estudios de neuroimágenes) capaces de detectar los supuestos desequilibrios bioquímicos (Wannmacher, 2006). La disminución de síntomas en los niños diagnosticados con TDAH cuando se les administra psicoestimulantes ha sido difundida como la forma de confirmar el diagnóstico (Mayes, Rafalovich, 2007). Sin embargo, como estos mismos autores muestran, Judith Rapaport, una de las primeras investigadoras en recibir fondos de los Institutos Nacionales de Salud de los Estados Unidos para investigar el TDAH, demostró ya en la década del setenta, que la administración de psicoestimulantes tenía similares efectos en niños diagnosticados y no diagnosticados con el trastorno.

A partir de la década del sesenta el gobierno federal de los Estados Unidos otorgó los primeros subsidios para investigación básica y epidemiológica sobre el TDAH con la finalidad de entender tanto la 
etiología del trastorno, como las dimensiones del problema, los grupos más afectados y los efectos de la medicación (Mayes, Rafalovich, 2007). Esto generó una mayor visibilidad del trastorno que poco a poco pasó de los ámbitos de la salud a los ámbitos educacionales desde donde se demandó reconocer a los niños diagnosticados con TDAH como discapacitados y elegibles para recibir educación especial. En 1991, el Departamento de Educación de los Estados Unidos implementó la ampliación del Acta de Discapacidades Individuales en la Educación (Individuals with Disabilities Education Act) para incluir a los niños con TDAH. Esto facilitó la aceptación social del TDAH dentro de las escuelas, así como un influjo de fondos y recursos humanos destinados a las mismas, ya que más niños fueron identificados para recibir educación especial (Davila, Williams, MacDonalt, 1991).

Los procesos descriptos anteriormente generaron un creciente aumento de niños diagnosticados y tratados en los Estados Unidos a partir de los noventas, fenómeno que se expande a otros países a partir de la década del 2000. América Latina no es la excepción; en esa región se puede observar una creciente proliferación de sitios en la red de asociaciones de profesionales, de familiares, de educadores y de empresas farmacéuticas que mimetizan los desarrollados en los Estados Unidos (ABDA, 2011; Fundación TDAH, 2011; Janssen-Cilag, 2010). En los últimos años, América Latina también ha visto el crecimiento de la divulgación del TDHA a través los medios de comunicación (Ortega et al., 2010). Asimismo, ha crecido el número de artículos sobre el trastorno publicados en revistas científicas latinoamericanas ${ }^{4}$. Las campañas de concientización sobre este trastorno financiadas por asociaciones de pacientes y de profesionales que se ocupan del tema y por las farmacéuticas que producen los medicamentos para el tratamiento del TDAH han aumentado en las escuelas, hospitales y otros ámbitos comunitarios (Faraone et al., 2010).

El argumento de quienes defienden la necesidad de que padres, educadores y la población en general se concienticen sobre este problema es que el aumento de casos diagnosticados y tratados muestra el éxito y la necesidad de estas campañas para identificar niños que padecen el trastorno (AAP, 2010). Este argumento, así como el difundido uso de los datos de prevalencia generados en los Estados Unidos para presentar el problema en otros países, pone de manifiesto la importancia de desarrollar un análisis crítico de cómo se construyen y reportan los datos de prevalencia del TDAH que presenta el CDC en su sitio de la red (CDC, 2011).

El CDC establece la prevalencia del TDAH en los Estados Unidos en base a la información recolectada en la Encuesta Nacional de Salud de los Niños (NSCH, por sus siglas en inglés). Esta encuesta se toma a una muestra al azar de números de teléfonos fijos. La encuesta es respondida por el adulto más informado sobre las condiciones de salud del niño seleccionado para integrar la muestra. La pregunta que se les hace en la encuesta telefónica es "si un médico u otro profesional de salud le ha informado alguna vez que su hijo tiene TDAH", si la respuesta es afirmativa, se les pregunta "si el niño actualmente toma medicación para el TDAH" (énfasis agregado).

La metodología, como el mismo CDC lo señala, tiene importantes limitaciones (Visser et al., 2010). Por un lado, se excluyen domicilios donde no hay teléfono fijo y aquellos entrevistados que no aceptan participar. Por otro, el tipo de preguntas supone la capacidad de los padres de recordar el diagnóstico, no confundirlo con otro, reconocer la severidad del problema y ser veraces en la respuesta. En la encuesta del 2007 se agregaron tres preguntas: "si el niño tiene en la actualidad TDAH", "la severidad del caso (leve, moderado, y severo)"; y "si el niño toma medicación para el trastorno".
${ }^{4}$ Las estadísticas que presenta Scielo, por ejemplo, al realizar una búsqueda usando la sigla TDHA o desglosándola, muestra que entre 1995 y 2003 el número de artículos que se encuentran llegan a un máximo de 6 en el 2003 en el 2004 aumentan a 16 y llegan a 31 en el 2010. Reconocemos que estas estadísticas no son exactas, pero nos permiten ver la creciente tendencia en el número de artículos publicados sobre el tema. 
La prevalencia de TDAH en la población infantil reportada en la página sobre este trastorno en el sitio del CDC corresponde a la encuesta del 2007 (Visser et al., 2010 ). Esta encuesta nacional recolectó datos de salud de 91.642 niños de 4 a 17 años de edad. El número de entrevistas con información completa en relación al TDAH fue de 63.123 casos. Usando las respuestas a la pregunta de si el niño alguna vez fue diagnosticado con TDAH, el CDC reporta en su página principal sobre el TDAH, un aumento del $22 \%$ de los casos entre el 2003 y el 2007, ya que las respuestas afirmativas a esa pregunta aumentaron del 7,8\% al 9,5\%. Sin embargo, si se considera la pregunta agregada en el 2007, de si el niño tenía el trastorno al momento de la entrevista, las respuestas positivas fueron considerablemente menos, ya que el 7,2\% de los niños padecían el trastorno según los entrevistados. Asimismo, de los niños cuyos padres informaron que tenían TDAH al momento de la entrevista, en casi la mitad de los casos $(46,7 \%$ ) la condición fue reportada como leve, en el $39,5 \%$ moderada y solo en el $13,8 \%$ de los casos severa. El $66,3 \%$ de los niños cuyos padres reportaron que tenían TDAH recibía medicación al momento de la entrevista, de los cuales, el $56,4 \%$ eran casos considerados leves, el $71,6 \%$ moderados, y el $85,9 \%$ severos.

Hasta aquí los datos que se pueden analizar e informar sobre el TDAH con la encuesta realizada en los Estados Unidos. Esto nos lleva a preguntarnos si es correcto que se priorice informar al público que hubo un aumento en la prevalencia de casos reportados por los padres del trastorno entre el 2003 y el 2007, como lo hace el CDC en su página y lo indica el título del artículo oficial donde se reportan los datos en mayor detalle. Usando la definición de prevalencia como el número de casos de una enfermedad o evento en una población en un momento dado, el dato que debería publicarse para el 2007 es el de 7,2\% (casos actuales de niños con el trastorno reportados por los padres). Asimismo, si el CDC quiere reportar alguna comparación con años anteriores debería aclarar que para propósitos comparativos se ven obligados a usar la pregunta de "si el niño alguna vez fue diagnosticado con TDAH" y no la agregada en el 2007 "de si el niño tenía el trastorno al momento de la entrevista" debido a la falta de información para esta pregunta en años anteriores.

Los medios de comunicación, aun los que ofrecen análisis críticos del problema, recurren a estos sitios generados por organismos gubernamentales cuando precisan información para encuadrar noticias o sus propias investigaciones sobre el TDAH (Democracy Now, 2011). Estos datos se toman como verdades incuestionables y facilitan la circulación de un discurso alarmista e incorrecto que se difunde por los medios de comunicación, y construye el sentido común alrededor de este problema. La utilización de generalizaciones estadísticas dificulta, por un lado, el entendimiento de las reales dimensiones del problema y, por el otro, favorece su rápida difusión. La creación de la preocupación colectiva alrededor de un tema lleva, muchas veces, a que los gobiernos inviertan fondos escasos en tratarlos o difundir información, que deberían destinarse a otros problemas que afectan a un número mayor de niños. Pero tal vez, lo más grave es que al facilitarse la difusión de la concepción del TDAH como una disfunción biológica del niño que puede ser tratada con medicación, se genera una sensación de alivio social ya que nadie es responsable y no hay nada que revisar a nivel de la vida social y familiar. Este alivio sería menor si se comprende cómo estos procesos favorecen la internacionalización del control y regulación de los cuerpos que responde a una forma de gobernabilidad biomédica. Gobernabilidad entendida en el sentido foucaultiano de formas particulares de poder, generalmente definidas por saberes especializados que implican monitorear, observar, medir, y normalizar individuos y poblaciones. Este poder no descansa en una coerción forzada, sino en mecanismos difusos, tal el caso de los discursos que prometen felicidad o salud a través de ciertas formas de conducta personal que requieren, entre otras cosas, autovigilancia y autorregulación (Clarke et al., 2010). En el caso del TDAH la promesa es un niño tranquilo, atento, y sociable que obtenga buenos resultados escolares y no problematice el espacio educativo, familiar y social. Por esto consideramos importante analizar a continuación como se realiza la construcción histórica y social de la definición y del diagnóstico del TDHA, ya que estas dos variables determinan la cantidad de casos diagnosticados y el tratamiento adoptado que conlleva a formas particulares de regulación social. 


\section{Antecedentes y subjetividad del diagnóstico}

La mayoría de los diagnósticos psiquiátricos se definen y redefinen en torno a un clima histórico sociocultural. De esta forma, diferentes categorías diagnósticas aparecen y desaparecen con el tiempo, lo cual refleja en muchos casos las ideologías científicas predominantes de la época. Las definiciones de los trastornos psiquiátricos no son necesariamente un indicador de condiciones clínicas objetivas, sino en muchos casos, el producto de un proceso influenciado primariamente por factores socioeconómicos, políticos, culturales, e ideológicos (Conrad, Potter, 2000; Cooksey, Brown, 1998).

En los Estados Unidos, la mayoría de los trastornos psiquiátricos se compilan en el Manual Diagnóstico y Estadístico (DSM por sus siglas en inglés), elaborado por la Asociación Americana de Psiquiatría (American Psychiatric Association, 2000). Este manual es el documento oficial donde se describen todos los cuadros psiquiátricos reconocidos en ese país por la ciencia hegemónica en un período específico. Esta guía es usada ampliamente por profesionales de la salud mental en sus prácticas clínicas, pero también por investigadores de diversas disciplinas. El uso de este manual diagnóstico se ha difundido internacionalmente en el campo de la psiquiatría, siendo en la actualidad más utilizado que la Clasificación Internacional de Enfermedades en su décima edición (CIE-10) (World Health Organization, 2009). Uno de los motivos por el cual el DSM se ha difundido tan ampliamente puede estar relacionado con el hecho de que las revistas de gran impacto científico en el campo de la salud mental requieren que los investigadores usen el DSM para que los manuscritos sean considerados para publicación (Dalal, Sivakumar, 2009). Asimismo, en los Estados Unidos y en otros países, la seguridad social médica y los seguros privados no pagan los servicios psicológicos o psiquiátricos a menos que el tratamiento esté incluido en un código diagnóstico del DSM aunque la persona consulte por problemas emocionales o relacionales no patológicos (Kutchins, Kirk, 1997).

Algunos autores argumentan que uno de los principales problemas del DSM es que fue desarrollado y es actualizado por una disciplina, la psiquiatría, que incluye diversas corrientes de pensamiento sustentadas en diferentes paradigmas, tales como: conductismo, psicoanálisis y neurobiología. Como resultado, la clasificación de los trastornos psiquiátricos varía dependiendo de la corriente de pensamiento que hegemonice la conceptualización de salud mental en un determinado período (Lee, 2002). Por ejemplo, las definiciones del TDAH han cambiado notablemente en las sucesivas revisiones del DSM desde su publicación en 1952. La caracterización del TDAH ha pasado de "infancia con neurosis" a "neurosis compulsiva" de acuerdo al discurso psicoanalítico, para luego transformarse en "ansiedad" de acuerdo al discurso psiquiátrico y finalmente se le ha definido como "trastorno de déficit de atención con o sin hiperactividad" según los discursos neurobiologistas (Rafalovich, 2005). Los diagnósticos basados en estos paradigmas tienden a desestimar las variaciones histórico-contextuales. Por ejemplo, en las últimas décadas se han producido profundos y acelerados cambios en todos los niveles de la vida individual y colectiva, que afectaron las condiciones de vida y trabajo de la mayoría de los grupos humanos y consecuentemente las formas en que las personas han respondido a estos cambios (Timimi, 2005). Sin embargo, la respuesta mayoritaria desde los servicios de salud a, por ejemplo, la ansiedad, la depresión, y la angustia generada por estos cambios ha sido la negación de estos como procesos sociales y su diagnóstico como desajustes individuales químico-biológicos que son tratados con fármacos (Jablensky, 1999). Esto ha exacerbado la respuesta medicalizadora a los problemas emocionales, relacionales y sociales en la mayoría de los países (Lee, 2002; Conrad, Potter, 2000).

Asimismo, varios estudios reportan diferencias claras entre la perspectiva europea y la estadounidense con respecto al diagnóstico del TDAH (Reid, Maag, 1997). Estas diferencias pueden deberse al uso de diferentes clasificaciones para realizar los diagnósticos, en Europa es más común el uso de la CIE-10, mientras que en los Estados Unidos se usa el DSM. El uso de la definición de TDAH de la última versión del DSM IV publicado en 1994, genera más casos diagnosticados de este trastorno que el uso de la definición de la CIE-10. Los criterios diagnósticos del DSM IV generan una prevalencia de este trastorno en la población general que varía entre 5\% y 10\%, mientras que usando los de la CIE-10 la prevalencia fluctúa entre $1 \%$ y $2 \%$ (Swanson et al., 1998). También se ha demostrado que las prevalencias de TDAH son más altas cuando se emplean los criterios diagnósticos del DSM-IV en 
comparación con las versiones anteriores del mismo manual (DSM III y DSM III-R), independientemente del país donde se haya llevado a cabo el estudio (Polanczyk et al., 2007). El diagnóstico del TDAH usando la CIE-10 es más restrictivo y requiere una mayor precisión sintomatológica (Tripp et al., 1999). La CIE-10 requiere que el niño muestre síntomas en las tres dimensiones del TDAH (inatención, hiperactividad e impulsividad) y estos síntomas deben observarse tanto en la escuela como en el hogar. En contraste, usando el DSM-IV se pueden diagnosticar con TDAH niños que muestran síntomas en una sola dimensión, por ejemplo, inatención, y requiere que solo alguno de los síntomas se presente tanto en la casa como en la escuela. Asimismo, el DSM-IV permite diagnosticar TDAH junto con otros trastornos psiquiátricos concomitantes, lo que no está aceptado por la CIE-10 (Moffitt, Melchior, 2007). También se ha reportado que la aplicación del criterio del DSM IV y su énfasis en los subtipos de TDAH parece incrementar la frecuencia con que se diagnostica este trastorno (Skounti, Philalithis, Galanakis, 2007).

A pesar de los avances científicos en las áreas de neurociencias, la mayoría de los diagnósticos psiquiátricos permanecen intrínsecamente ligados al juicio subjetivo del clínico y a la forma en que el paciente pueda comunicar sus síntomas (Strauss, 1996). Asimismo, los criterios del DSM-IV no toman en cuenta diferencias de género, socioculturales o variaciones en las etapas del desarrollo de los niños, lo que puede llevar a diferentes observadores a interpretar el mismo comportamiento de manera distinta (Rohde et al., 2005). Jablensky (1999) argumenta que el DSM está basado en la suposición de que los trastornos psiquiátricos forman categorías discretas. El DSM requiere juicios categóricos de "si/ no" con respecto a si el paciente muestra un comportamiento específico de los síntomas listados para un determinado trastorno. Esto dificulta la consideración de relaciones complejas entre los aspectos culturales y sociales con relación a la salud mental (Mezzich, Fabrega Junior, Kleinman, 1992). La evidencia que se requiere para hacer un diagnóstico con el DSM es primariamente fenomenológica y de descripción del comportamiento.

Otro elemento controversial del DSM-IV son los lazos financieros de sus autores con la industria farmacéutica. Un estudio publicado en 2006 demostró que de los 170 miembros del panel del DSM, 95 (56\%) tenían uno o más lazos financieros con la industria farmacéutica. El estudio indica que las relaciones financieras entre los autores del DSM-IV y las compañías farmacéuticas son especialmente fuertes en aquellas áreas diagnósticas donde los medicamentos son la primera línea de tratamiento. (Cosgrove et al., 2006) Asimismo, la industria farmacéutica ha sido eficaz en convencer a los seguros médicos de que los tratamientos con medicamentos son menos costosos que las terapias psicológicas y los ha llevado en muchos casos a negar este tipo de cobertura a sus asegurados (Horwitz, 2010).

Hasta aquí hemos analizado que tanto los datos de prevalencia del TDAH como las definiciones del trastorno usadas para el diagnóstico, no son elementos objetivos o verdades inmutables en el tiempo y espacio. Estas son construcciones elaboradas por determinados grupos profesionales e investigadores influenciados por el discurso científico que hegemoniza una época, país o región y que se instala como la verdad acerca de un saber en salud. Asimismo, las definiciones de las categorías diagnósticas están, en muchos casos, influenciadas por los intereses económicos y las relaciones financieras que los grupos que lideran las organizaciones profesionales, así como las de pacientes, mantienen con el complejo médico-industrial. El problema es que el común de la población, muchos investigadores y profesionales de salud desconocen estos procesos y el discurso hegemónico se transforma en la verdad sobre el saber en salud, en este caso en torno al TDAH. Esto legitima las formas diagnósticas, los tratamientos, y la construcción y circulación de los datos estadísticos, así como la creación de nuevas biosocialidades.

\section{Conclusiones}

En este artículo presentamos un análisis que contextualiza la problemática del TDAH en los procesos de reacomodamiento que el complejo médico-industrial ha realizado para mantener o acrecentar su participación económica. Estos procesos los encuadramos en la profundización de la medicalización, definida como biomedicalización y analizamos cómo la forma en que el problema del TDAH se define, cuantifica y presenta al público contribuye a la biomedicalización de la infancia. 
No pretendemos proveer respuestas unívocas, sino reinterpretar la situación conectando diversos procesos que aparecen desconectados en torno a la problemática del TDAH. Para esto realizamos una relectura de las transformaciones desplegadas por la industria farmacéutica para revitalizar su papel en el liderazgo del complejo médico-industrial y en el mercado de salud, a la luz de las modalidades que adquieren la construcción y utilización de los datos estadísticos, la difusión de la información, la elaboración de los manuales diagnósticos, y otros discursos que intervienen en la producción y reproducción del sentido común en torno al TDAH. Con estos análisis esperamos ayudar a contextualizar estos fenómenos en el intenso proceso de gobernabilidad biomédica que supone la internalización del control y la regulación de los cuerpos. Estos procesos están en pleno desarrollo en el campo sanitario y afectan la vida de millones de seres humanos con resultados impredecibles. Asimismo, deseamos contribuir a expandir el pensamiento crítico sobre el TDAH, favoreciendo la multiplicación de voces que analizan el problema desde distintos ángulos. Numerosos investigadores y profesionales de salud están creando y difundiendo información que ofrece otras miradas sobre estos y otros padecimientos catalogados como problemas de salud mental. La biomedicalización del sufrimiento infantil facilita el encubrimiento de los profundos cambios socioeconómicos, políticos, e ideológico/ culturales que han transformado radicalmente nuestras sociedades en las últimas décadas. Como sociedad, deberíamos preguntarnos si las conductas disruptivas y antisociales de algunos niños no nos están alertando de un malestar con una forma de producción y reproducción de las sociedades donde, por un lado se les ofrece más posibilidades tecnológicas y educativas, pero por otro se los aliena desde muy pequeños de lo lúdico, lo solidario, y lo afectivo. Cerramos este artículo parafraseando la respuesta de un paciente de 10 años a su médico: El TDAH no es un problema de desbalance químico, es un problema de desbalance en las formas de vida (Miller, 1999 p.78).

\section{Colaboradores}

Las dos autoras, Celia Iriart y Lisbeth Iglesias Ríos participaron igualmente de la elaboración del artículo en lo que hace a revisiones bibliográficas, análisis y redacción del manuscrito. Celia Iriart contribuyó con el diseño del estudio, y análisis e interpretación de fuentes de información primarias y secundarias. Las dos autoras leyeron y aprobaron la versión final.

\section{Referencias}

AMERICAN ACADEMY OF PEDIATRICS - AAP. ADHD. Disponible en: <http://www.aap.org/healthtopics/adhd.cfm>. Acceso en: 30 nov. 2010.

AMERICAN PSYCHIATRIC ASSOCIATION - APA. Diagnostic and statistical manual of mental disorders. Arlington: American Psychiatric Association, 2000.

ASSOCIAÇÃO BRASILEIRA DO DÉFICIT DE ATENÇÃO - ABDA. Disponible en: $<$ http://www.tdah.org.br/>. Accesso en: 9 mayo 2011.

CENTERS FOR DISEASE CONTROL AND PREVENTION - CDC. Attention Deficient/ Hyperactivity Disorder (ADHD). Disponible en: <http://www.cdc.gov/ncbddd/adhd/ data.html>. Acceso en: 23 abr. 2011.

CHILDREN AND ADULTS WITH ATTENTION-DEFICIT/HYPERACTIVITY DISORDER CHADD. Welcome to CHADD's e-communities. Disponible en: <http:// www.chadd.org/AM/

Template.cfm?Section=Online Communities Guidelines\&Template $=/ \mathrm{CM} /$ HTMLDisplay.cfm\&contentID $=\overline{9} 970>$. Acceso en: 9 mayo 2011. 
CLARKE, A. et al. Biomedicalization: a substantive introduction. En: (Eds.). Biomedicalization: technoscience, health and illnesses in the U.S. London: Duke University Press, 2010. p.1-44.

CONRAD, P.; POTTER, D. From hyperactive children to ADHD adults: observations on the expansion of medical categories. Soc. Probl., v.47, n.4, p.559-82, 2000.

COOKSEY, E.; BROWN, P. Spinning on its axes: DSM and the social construction of psychiatric diagnosis. Int. J. Health Serv., v.28, n.3, p.525-54, 1998.

COSGROVE, L. et al. Financial ties between DSM-IV panel members and the pharmaceutical industry. Psychother. Psychosom., v.75, n.3, p.154-60, 2006.

CRITSER, G. Generation RX: how prescription drugs are altering American lives, minds, and bodies. New York: Houghton Mifflin Co., 2005.

DALAL, P.; SIVAKUMAR, T. Moving towards ICD-11 and DSM-V: Concept and evolution of psychiatric classification. Indian J. Psychiatry., v.51, n.4, p.310-9, 2009

DAVILA, R.R.; WILLIAMS, M.L.; MAC DONALT, J.T. Clarification of policy to address the needs of children with attention deficit disorder within general and/or special education. Washington: Department of Education, 1991.

DEATH FROM RITALIN. The truth behind ADHD. Disponible en: <http://www. ritalindeath.com >. Acceso en: 9 mayo 2011.

DEMOCRACY NOW! THE WAR AND PEACE REPORT. Dr. Gabor Maté on ADHD, bullying and the destruction of American childhood. Disponible en: <http:// www.democracynow.org/2010/11/24/dr_gabor_mat_on_adhd_bullying >. Acceso en: 25 mar. 2011.

FARAONE, S. et al. Discurso médico y estrategias de marketing de la industria farmacéutica en los procesos de medicación de la infancia en Argentina. Interface Comunic., Saude, Educ., v.14, n.34, p.483-97, 2010.

FRENK MORA, J. et al. Programa Específico de Trastorno por Déficit de Atención 2001-2006. Mexico City: Secretaria de Salud, 2002. p.1-110.

FUNDACIÓN TDAH. Educar para cambiar atendiendo la diversidad. Argentina: FUNDACIÓN Trastorno por Déficit de Atención e Hiperactividad TDAH, 2005-2009. Disponible en: <http://www.tdah.org.ar/>. Acceso en: 9 mayo 2011.

HORWITZ, A. Pharmaceuticals and the medicalization of social life. En: LIGHT, D. (Org.). The risks of prescription drugs. New York: Columbia University Press, 2010.

ILLICH, I. Medical nemesis: the expropriation of health. New York: Penguin,1975.

IRIART, C.; FRANCO, T.; MERHY, E.E. The creation of the health consumer: challenges on health sector regulation after managed care era. Globalization Health, v.7, n.2, p.1-12, 2011. Disponible en: <http://www.globalizationandhealth.com/content/7/1/ 2>. Acceso en: 18 ene. 2011.

IRIART, C.; MERHY, E.E.; WAITZKIN, H. La atención gerenciada en América Latina. Transnacionalización del sector salud en el contexto de la reforma.Cad. Saude Publica, v.16, n.1, p.95-105, 2000.

JABLENSKY, A. The nature of psychiatric classification: issues beyond ICD-10 and DSM-IV. Aust. N. Z. J. Psychiatr., v.33, n.2, p.137-44, 1999.

JANSSEN-CILAG. TDAH: manual para padres. Disponible en: <http://www. trastornohiperactividad.com/>. Acceso en: 1 dic. 2010.

KUTCHINS, H.; KIRK, S. Making us crazy: DSM - the psychiatric bible and the creation of mental disorders. New York: The Free Press, 1997.

LEE, S. Socio-cultural and global health perspectives for the development of future psychiatric diagnostic systems. Psychopathology, v.35, n.2-3, p.152-7, 2002. 
MAYES, R.; RAFALOVICH, A. Suffer the restless children: the evolution of ADHD and paediatric stimulant use, 1900-80. Hist. Psychiatr., v.18, n.72, p.435-57, 2007.

MEZZICH, J.; FABREGA JÚNIOR, H.; KLEINMAN, A. Cultural validity and DSM-IV. J. Nerv. Ment. Dis., v.180, n.1, p.4, 1992.

MILLER, L. Running on ritalin: a physician reflects on children, society and performance in a pill. New York: Bantam Books,1999.

MOFFITT, T.; MELCHIOR, M. Why does the worldwide prevalence of childhood attention deficit hyperactivity disorder matter? Am. J. Psychiatry, v.164, n.6, p.856-8, 2007.

MOYNIHAN, R.; CASSELS, A. Selling sickness: how the world's biggest pharmaceutical companies are turning us all into patients. New York: Penguin, 2005.

ORTEGA, F. Biopolíticas da saúde: reflexões a partir de Michel Foucault, Agnes Heller e Hannah Arendt. Interface - Comunic., Saude, Educ., v.8, n.14, p.9-20, 2003-2004.

ORTEGA, F. et al. A ritalina no Brasil: produções, discursos e práticas. Interface Comunic., Saude, Educ., v.14, n.34, p.499-510, 2010.

POLANCZYK, G. et al. The worldwide prevalence of ADHD: a systematic review and metaregression analysis. Am. J. Psychiatry, v.164, n.6, p.942-8, 2007.

RABINOW, P. Artificiality and enlightenment. En: CRARY, J.; KWINTER, S. (Orgs.). Incorporations. New York: Zone, 1995. p.234-52.

RAFALOVICH, A. Exploring clinician uncertainty in the diagnosis and treatment of attention deficit hyperactivity disorder. Sociol. Health Illn., v.27, n.3, p.305-23, 2005.

REID, R.; MAAG, J. Attention Deficit Hyperactivity Disorder: over here and over there. Educ. Child Psychol., v.14, n.1, p.10-20, 1997.

ROHDE, L.A. et al. Attention-deficit/hyperactivity disorder in a diverse culture: do research and clinical findings support the notion of a cultural construct for the disorder? Biol. Psychiatry, v.57, n.11, p.1436-41, 2005.

SKOUNTI, M.; PHILALITHIS, A.; GALANAKIS, E. Variations in prevalence of attention deficit hyperactivity disorder worldwide. Eur. J. Pediatr., v.166, n.2, p.117-23, 2007.

STRAUSS, J. Subjectivity. J. Nerv. Ment. Dis., v.184, n.4, p.205-12, 1996.

SWANSON, J. et al. Attention-deficit hyperactivity disorder and hyperkinetic disorder. Lancet, v.351, n.9100, p.429-33, 1998.

THE NATURAL CHILD PROJECT. Does ADHD even exist? The ritalin sham. Disponible en: <http://naturalchild.org/guest/john_breeding.html>. Acceso en: 9 mayo 2011.

TIMIMI, S. Effect of globalisation on children's mental health. Br. Med. J., v.331, n.7507, p.37-9, 2005.

TRIPP, G. et al. DSM-IV and ICD-10: a comparison of the correlates of ADHD and hyperkinetic disorder. J. Am. Acad. Child Adolesc. Psychiatry, v.38, n.2, p.156-64, 1999.

VISSER, S. et al. Increasing prevalence of parent-reported Attention-Deficit/ Hyperactivity Disorder among children. United States, 2003 and 2007. Morb. Mortal. Wkly Rep. (MMWR), v.59, n.44, p.1439-43, 2010.

WANNMACHER, L. DHDA: correto diagnóstico para real indicação de psicoestimulantes. Uso Racional de Medicamentos: Temas Seleccionados. OPS/OMS, v.3, n.10, p.1-6, 2006. Disponible en: <http://portal.saude.gov.br/portal/arquivos/ pdf/v3n10_dhda_psicoestimulantes.pdf>. Acceso en: 16 nov. 2010.

WORLD HEALTH ORGANIZATION - WHO. International Classification of Diseases (ICD). Disponible en: <http://www.who.int/classifications/icd/en/>. Acceso en: 23 dic. 2009. 
IRIART, C.; IGLISIAS RÍOS, L.

IRIART, C.; IGLESIAS RÍOS, L. Biomedicalização e infância: transtorno de déficit de atenção de hiperatividade. Interface - Comunic., Saude, Educ., v.16, n.43, p.1011-23, out./dez. 2012.

$\mathrm{O}$ artigo analisa criticamente o aumento das crianças diagnosticadas e tratadas por Transtorno de Déficit de Atenção de Hiperatividade (TDAH). As análises vinculam este crescente fenômeno às estratégias da indústria farmacêutica para se reposicionarem na liderança da conceituação do processo saúde-doença-atenção e no mercado de saúde. Utilizamos métodos analítico-interpretativos para estudar dados primários e secundários, e realizar uma extensa revisão bibliográfica. À luz do conceito da biomedicalização, analisamos os mecanismos subjetivo-ideológicos que facilitaram que este discurso se institua como uma nova verdade sobre este transtorno e seja legitimado pelos organismos governamentais e organizações da sociedade civil. A biomedicalização do sofrimento infantil dificulta que se revelem as profundas mudanças socioeconômicas, políticas e ideológico-culturais que têm transformado radicalmente nossas sociedades nas últimas décadas.

Palavras-chave: Transtorno de déficit de atenção com hiperatividade (TDAH). Biomedicalização. Consumidor de saúde. DSM. Complexo médico-industrial. Uso de medicamentos.

Recebido em 29/01/12. Aprovado em 07/07/12. 
Ricardo Pozzo, Ocupação Nova Primavera-CIC/Sabará, 2012

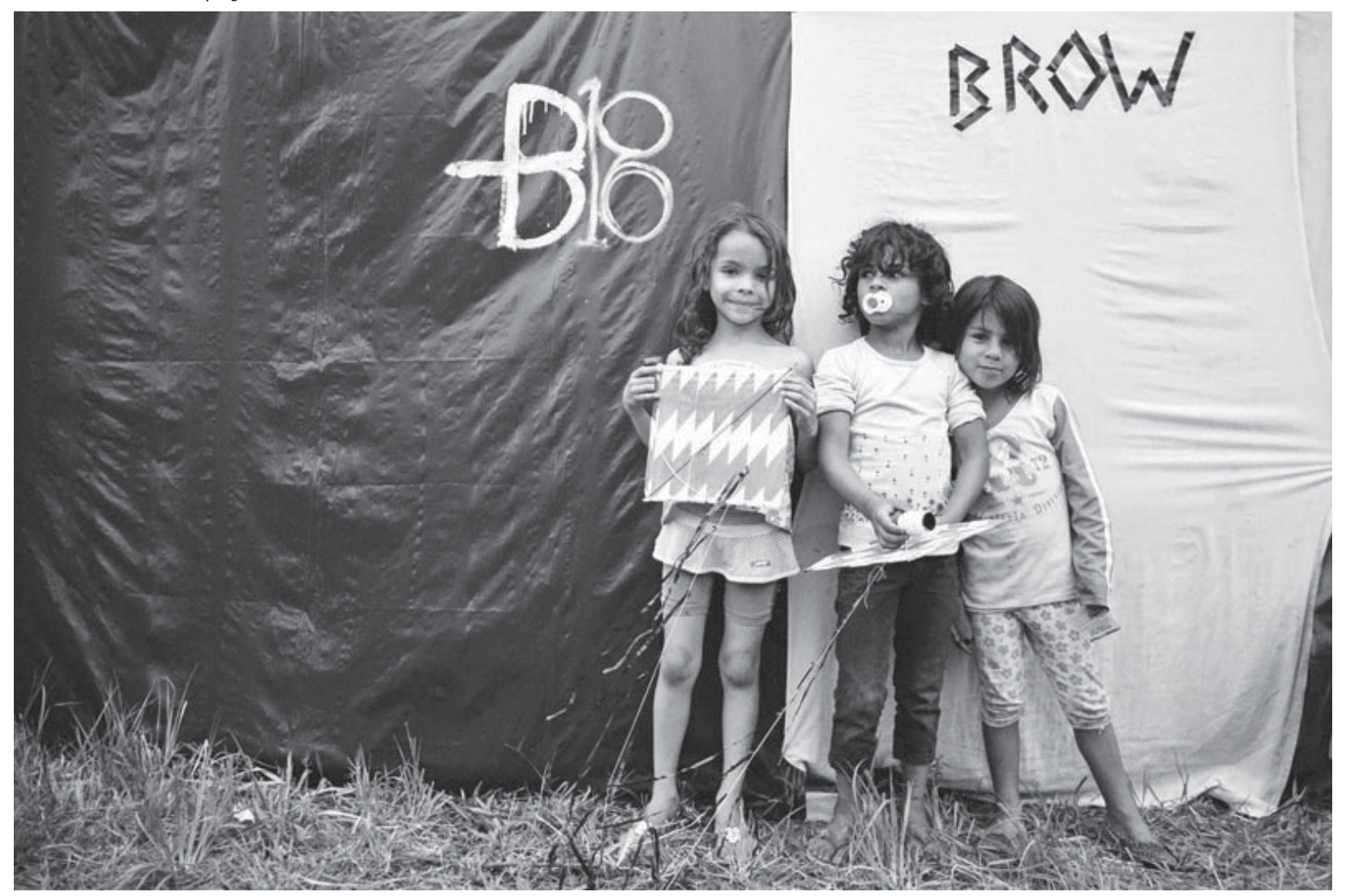

